\section{Enterocolite necrosante em recém-nascidos de muito baixo peso em uma unidade neonatal de alto risco do Nordeste do Brasil (2003-2007)}

\section{Necrotizing enterocolitis in very low weight newborns at a high risk neonatal unit in the Northeast region of Brazil (2003-2007)}

Taciana Duque Braga 1 Giselia Alves Pontes da Silva 2 Pedro Israel Carvalho Lira 3 Marília de Carvalho Lima 4
1 Faculdade Pernambucana de Saúde. Rua Jean Émile Favre, 422. Imbiribeira. Recife, PE, Brasil. CEP: 51.200-060. E-mail: tacianaduque@fps.edu.br 2-4 Universidade Federal de Pernambuco. Recife, PE, Brasil.

\begin{abstract}
Objectives: to assess the incidence of nectrotizing enterocolitis (NEC), between 2003 and 2007, in children born with a very low weight and to describe the care provided for these newborns.

Methods: a retrospective longitudinal study was carried out at a high-risk neonatal unit with a sample of 1028 newborns with very low birth weight, excluding those weighing less than $750 \mathrm{~g}$. The frequency of NEC was assessed for the first to thirtieth day of life, or until the disease appeared or the child died, considering the first occurrence in a period of five years. NEC was defined according to Bell's criterion (stage $\geq 2$ ). The chi-squared test was used to assess the behavior of the category variables over the five years.

Results: NEC occurred in 55 of the 1028 newborns $(5.3 \%)$. The highest incidence was found in the year 2005 (19/204) and there was a significant decrease in 2007 (8/195), although this variation could not be considered a trend $(p=0.45)$. A significant increase was found in the use of antibiotics, parenteral feeding, antenatal corticoid and the consumption of mother's milk, along with a decrease in the mean gestational age of 2.4 weeks between 2003 and 2007.

Conclusions: the incidence of NEC varied, showing no tendency to increase or decrease. There was an increase in antenatal corticosteroids coverage, breast milk intake and a reduction in the use of formula. Further studies are needed to provide a better evaluation of these measures in the nonprogression of incidence of NEC, even though this is a population that is highly at risk from the contracting the disease.
\end{abstract}

Key words Necrotizing enterocolitis, Infant, very low birth weight

\section{Resumo}

Objetivos: avaliar a incidência de enterocolite necrosante (ECN), no periodo de 2003 a 2007, em crianças nascidas com muito baixo peso e descrever as características da assistência prestada a esses recém-nascidos.

Métodos: estudo longitudinal retrospectivo conduzido em uma unidade neonatal de alto risco com uma amostra de 1028 recém-nascidosde muito baixo peso, excluindo-se os menores de $750 \mathrm{~g}$. A frequência de ECN foi observada do primeiro até o trigésimo dia de vida, ou até a sua ocorrência ou óbito, considerando-se o que ocorresse primeiro em um periodo de cinco anos. ECN foi definida pelo critério de Bell (estágio $\geq 2$ ). Utilizou-se o quiquadrado de tendência para avaliar o comportamento das variáveis categóricas ao longo dos cinco anos.

Resultados: ECN ocorreu em 55 dos 1028 recémnascidos (5,3\%). Observou-se maior incidência de ECN no ano de 2005 (19/204) com uma significante redução no ano de 2007 (8/195), entretanto, essa variação não caracterizou uma tendência $(p=0,45)$. Observou-se aumento significante do uso de antibióticos, de nutrição parenteral, de corticóide antenatal e do consumo de leite materno e uma redução na média da idade gestacional de 2,4 semanas entre 2003 e 2007.

Conclusões: a incidência de ECN foi variável, sem tendência de ascensão ou queda. Observou-se um incremento na cobertura de corticoide antenatal, aumento na utilização de leite materno e redução no consumo de fórmula. Mais estudos são necessários para melhor avaliar a contribuição dessas medidas na não progressão da incidência da ECN, apesar de tratar-se de uma população com risco elevado para essa doença.

Palavras-chave Enterocolite necrosante, Recémnascido de muito baixo peso 


\section{Introdução}

Enterocolite necrosante (ECN) é uma importante causa de morbiletalidade em prematuros, sendo uma das mais graves complicações relacionada ao tubo digestório nesse grupo de crianças. Apresenta uma incidência variável e inversamente proporcional à idade gestacional. Considera-se que cerca de $12 \%$ das crianças de muito baixo peso (peso de nascimento abaixo de $1500 \mathrm{~g}$ ) podem ser atingidas por essa doença, e dessas, $30 \%$ ou mais poderão evoluir para o óbito.1,2

A patogênese da ECN é multifatorial e complexa; a prematuridade é considerada o principal fator de risco para a sua ocorrência, 3,4 e as medidas de prevenção ainda não são consistentemente efetivas. 1,3-6

A ocorrência de ECN é preocupante, pois, na maioria das vezes, acomete recém-nascidos que sobreviveram aos agravos do período neonatal precoce. ${ }^{1}$ A magnitude desse problema fica mais evidente quando se observa que, mesmo aqueles que sobrevivem à doença, podem apresentar evolução desfavorável relacionada tanto a complicações póscirúrgicas quanto aos desenvolvimentos neurológico, visual e auditivo. 1,3

Apesar de a ECN ser descrita como de ocorrência esporádica, existem algumas publicações que relatam prováveis surtos dessa doença.7,8 Os critérios utilizados pela maioria dos autores para a definição desses surtos são questionados, pois os períodos relatados como de maior incidência podem ser decorrentes da variação natural na ocorrência da doença. Por outro lado, observa-se que muitos serviços que relatam baixas taxas de ECN não apresentam o comportamento histórico dessa incidência, e os dados relacionados a um único período podem não traduzir a situação da ECN para aquela determinada Unidade de Terapia Intensiva Neonatal. ${ }^{9}$

Este estudo tem como objetivo avaliar a incidência de ECN com análise de tendência, no período de 2003 a 2007, em crianças nascidas com muito baixo peso em uma unidade neonatal de alto risco e descrever as características da assistência prestada a esses recém-nascidos.

\section{Métodos}

O estudo foi realizado na Unidade Neonatal do Instituto de Medicina Integral Professor Fernando Figueira (IMIP), um hospital terciário de ensino e pesquisa da cidade do Recife, Pernambuco, de referência ao atendimento de gestantes de alto risco, com uma média de 460 partos ao mês. A unidade neonatal possui 18 leitos de UTI e 32 leitos de cuidados semiintensivos, destina-se exclusivamente ao atendimento das crianças nascidas na instituição.

Foram incluídos no estudo recém-nascidos com peso de nascimento entre $750 \mathrm{~g}$ e $1500 \mathrm{~g}$, a faixa de peso de maior ocorrência de ECN segundo estudo piloto prévio, admitidos na unidade neonatal de alto risco no período de janeiro de 2003 a dezembro de 2007. Excluíram-se a ocorrência de óbito nas primeiras 24 horas de vida, os que apresentaram malformações congênitas graves relacionadas ao trato digestório, síndromes genéticas e infecções congênitas diagnosticadas ao nascimento.

Realizou-se estudo longitudinal, retrospectivo, no qual foram analisadas a frequência de $\mathrm{ECN}$ e as caracteristicas gerais dos recém-nascidos e da assistencia a eles prestada em um período de cinco anos.

A coleta de dados foi realizada através da análise de prontuários, sendo preenchidos formulários estruturados para registros diários das características biológicas dos recém-nascidos ( $\mathrm{RN}$ ), da assistência pré-natal, natal, e da assistência pós-natal desde o primeiro até o trigésimo dia de vida, ou até a ocorrência de ECN ou óbito, considerando o que ocorreu primeiro. Os casos de ECN foram definidos pela descrição em prontuário de distensão abdominal e pneumatose intestinal ao exame radiológico de abdome (estágio $\geq 2$ pelo critério de Bell10) definido pelo neonatologista e confirmado pelo laudo do radiologista. $\mathrm{O}$ peso ao nascer foi registrado segundo a rotina do serviço, com mensuração no momento do nascimento, utilizando-se balança eletrônica marca Filizola, modelo $B P \mathrm{n}^{\circ} 620 / 95$, com capacidade para $15 \mathrm{Kg}$, e da idade gestacional pelo método de Ballard. ${ }^{11}$ A relação das crianças nascidas no período foi obtida por consulta à base de dados do Sistema de Nascidos Vivos (SINASC), através do núcleo de epidemiologia da instituição.

O processamento e a análise dos dados foram rea-lizados no pacote estatístico Epi-Info, versão 6.04 (CDC, Atlanta) e no Statistical Package for the Social Sciences (SPSS Inc., Chicago, Ill), versão 12.0. Utilizou-se o teste do qui-quadrado de tendência para avaliar o comportamento das variáveis categóricas ao longo dos cinco anos. $\mathrm{O}$ teste $t$ de Student foi empregado para comparar as diferenças de média entre as variáveis contínuas, e o qui-quadrado, para as diferenças de proporção entre as variáveis categóricas, empregando-se o teste exato de Fisher, quando indicado. Adotou-se o valor de $p \leq 0,05$ como nível de significância estatística para todas as análises.

Esse estudo foi aprovado pelo Comitê de Ética em Pesquisa em Seres Humanos do IMIP. 


\section{Resultados}

Foram analisados 1028 prontuários de crianças admitidas na UTIN com peso entre $750 \mathrm{~g}$ e $1500 \mathrm{~g}$ no período do estudo. ECN foi observada em 55 $(5,3 \%)$,e dessas, 20 (36\%) evoluíram para o óbito. A ocorrência de ECN por ano estudado é apresentada na Figura 1. Observou-se maior incidência de ECN no ano de $2005(p=0,03)$ mas não foi identificada nenhuma tendencia de ascensão ou queda na ocorrência desse desfecho durante o período do estudo $\left(\chi^{2}\right.$ de tendência $\left.-p=0,45\right)$.

$\mathrm{Na}$ Tabela 1, estão registradas algumas características desses $\mathrm{RN}$ e os cuidados pré e pós-natais, a cada ano. Não houve diferença entre a média de peso ao nascer, mas observou-se idade gestacional mais baixa nos últimos anos analisados $(p<0,001)$. Em relação aos cuidados perinatais durante os cinco anos, observou-se tendência de aumento do uso de corticóide antenatal, do uso de antibióticos e do uso de nutrição parenteral total (NPT) $(p<0,001)$. Em relação à dieta enteral houve uma tendência de aumento do uso de leite materno exclusivo por mais de sete dias e tendência de redução no uso exclusivo de fórmula por mais de um dia $(p<0,001)$.

\section{Discussão}

A ECN é uma doença de ocorrência pós-natal com considerável variação de incidência entre os diversos serviços. Ocorre de forma predominante em prematuros, principalmente, naqueles com peso de nascimento inferior a 1500 g.4,12,13 Para melhor compreensão do comportamento da $\mathrm{ECN}$, foram analisados 1028 recém-nascido de muito baixo peso (RNMBP), admitidos em uma UTI neonatal que possui rotinas assistenciais e critérios diagnósticos padronizados. O serviço estudado é referência regional para gestações de alto risco, o que favoreceu a avaliação de um número elevado de RNMBP, mesmo tratando-se de estudo unicêntrico.

Apesar de tratar-se de estudo retrospectivo com coleta de dados através dos prontuários, observou-se um bom preenchimento das informações, provavelmente por tratar-se de um hospital de ensino e possuir um bom monitoramento das condições de morbidade desses recém-nascidos. Foi encontrada uma frequência de $5,3 \%$, com variações entre os anos para a ECN. A maioria dos relatos de incidência de ECN são geralmente relacionados a períodos curtos sem análise de tendência nos diversos serviços. Relatório da Rede Brasileira de pesquisa Neonatal mostra uma incidência de $6,0 \%$ relativa ao

\section{Figura 1}

Enterocolite necrosante em recém-nascidos de muito baixo peso.

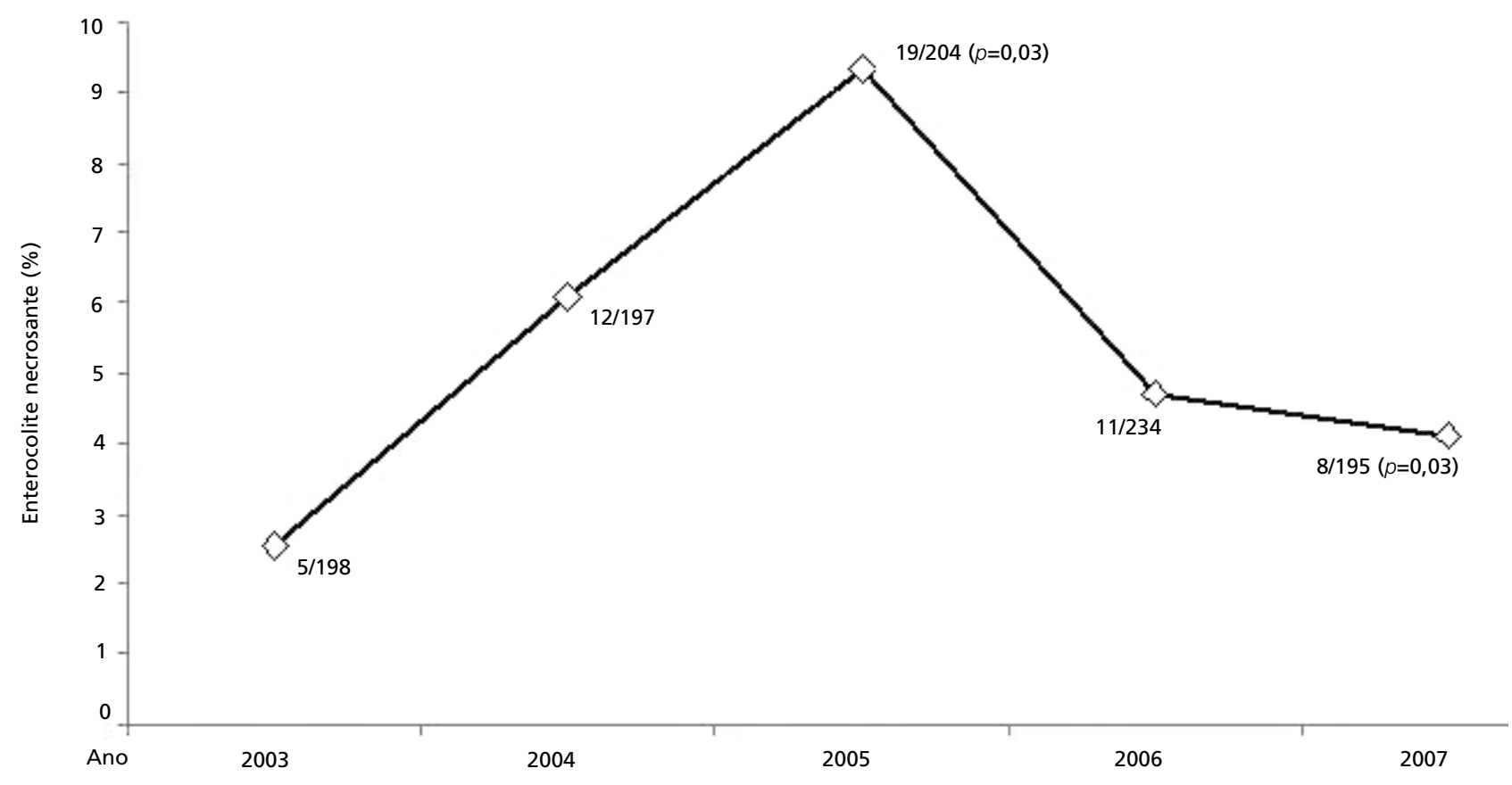

Análise de tendência: $\chi^{2}$ de tendência; $p=0,45$.

Fonte: IMIP, 2003 a 2007. 


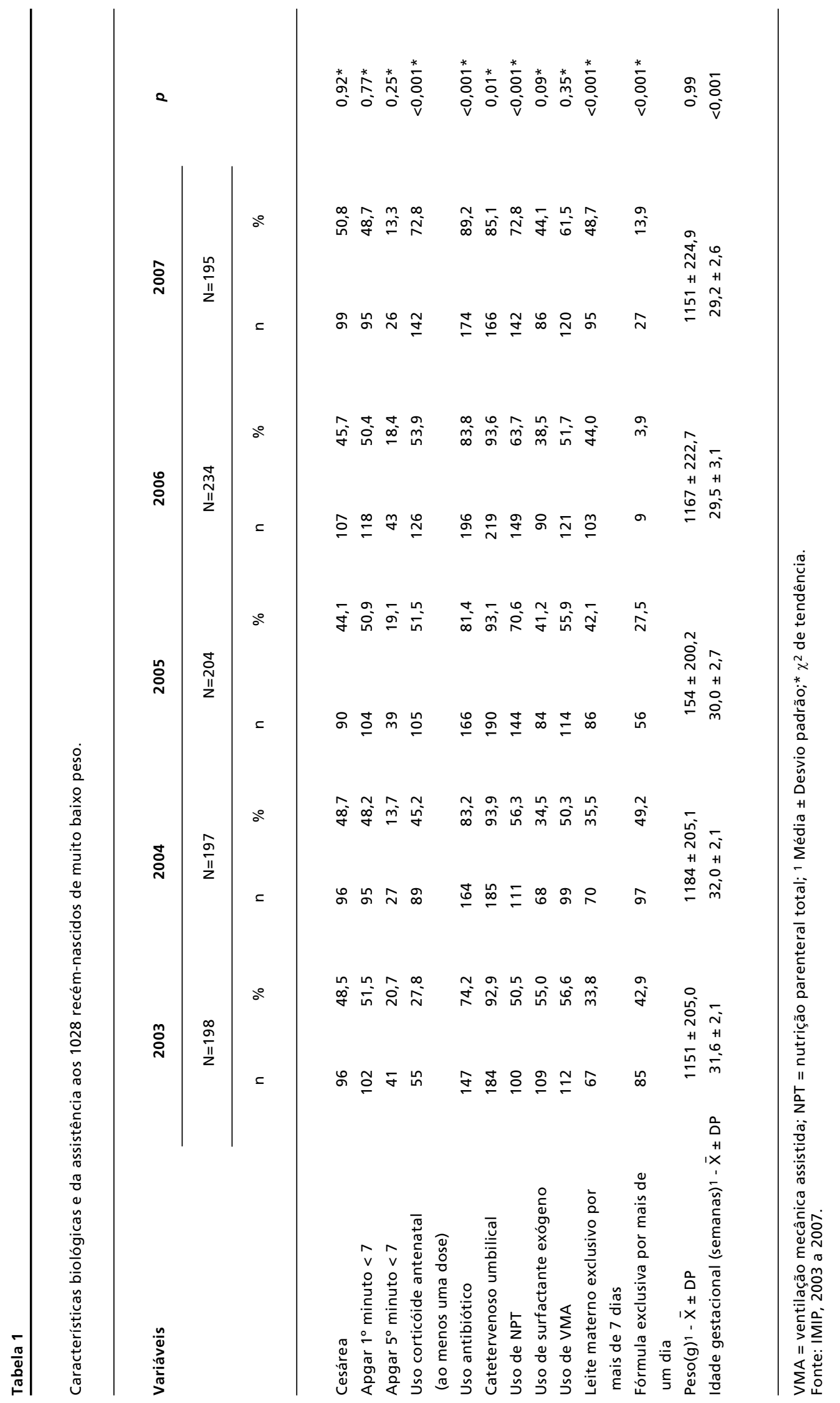


ano de 2010.14

A variação nos critérios diagnósticos é outra dificuldade na interpretação dos resultados nos diversos estudos.7,9,14 Incluímos como caso apenas aqueles que apresentavam ao raio $\mathrm{X}$ de abdome pneumatose intestinal (Estágio $\geq 2$ pelo critério de Bell), evitando-se a dúvida nos diagnósticos utilizando-se outros critérios, em que quadros como a perfuração intestinal focal (PIF) podem ser erroneamente incluídos. ${ }^{15} \mathrm{O}$ presente estudo mostrou uma variação no número de casos a cada ano, entretanto, apesar da redução na frequência de ECN nos dois últimos anos (2006 e 2007), esse fato não caracterizou uma tendência. Período mais longo de observação pode ser necessário para melhor compreensão do comportamento da ECN no serviço.

Em relação às características dos $\mathrm{RN}$ ao longo dos cinco anos, observou-se redução na média da idade gestacional. É sabido que a prematuridade é considerada o principal fator de risco para a ocorrência de ECN.1,16,17 O aumento dessa susceptibilidade é atribuído ao grau de imaturidade intestinal relacionado às funções motora e digestiva, integridade e função da defesa mucosa local, regulação da resposta inflamatória gastrointestinal, respostas hemodinâmicas das circulações esplâncnicas e sistêmicas, associadas à presença do substrato na luz do intestino e modificação no padrão de colonização intestinal. 1,18

Especula-se que a idade gestacional mais baixa associada à tendência de aumento do uso de antibióticos e de nutrição parenteral possa traduzir uma população de risco ascendente para a ocorrência de ECN. O uso de antimicrobianos interfere no padrão de colonização intestinal do recém-nascido, pois leva à supressão de bactérias anaeróbicas com consequente desequilíbrio da microflora endógena, podendo esse desequilíbrio ser transitório, ou prolongar-se por algum período após o seu uso. 16,18-20 As características da microflora endógena do prematuro vêm sendo apontadas em diversos estudos como fator de grande importância para a cascata de eventos que culminam com a ocorrência da ECN.18,21,22 A maioria dos RNMBP apresenta co-morbidades, impedindo ou retardando o início da dieta por via enteral, com necessidade da utilização de nutrição parenteral.

Por outro lado, rotinas assistenciais descritas como protetoras em relação à ocorrência da ECN foram implementadas ao longo dos cinco anos, como aumento do uso do corticóide ante natal, redução no consumo de fórmula e maior tempo de utilização de leite materno exclusivo. Estudos clínicos têm mostrado que o uso antenatal de corticóides reduz a incidência de ECN.1,4,16,20 O fator de ativação plaquetária (FAP) é um mediador pró-inflamatório da injúria intestinal, cujo efeito sistêmico em estudos animais é similar à apresentação clínica de crianças com ECN grave e inclui choque, isquemia e necrose intestinal, mostrando ser um importante mediador inflamatório na fisiopatologia da doença. A FAPacetilhidrolase é a enzima responsável pela degradação do fator de ativação plaquetária, apresentando níveis baixos ao nascimento, só atingindo os níveis do adulto a partir da $6^{\mathrm{a}}$ semana de vida. A dexametasona estimula a atividade da FAP-acetil hidrolase, o que pode explicar o efeito protetor do corticóide antenatal para a ECN.18,21,23,24 Acreditase, ainda, que o padrão de motilidade intestinal do prematuro favoreça o supercrescimento bacteriano, culminando com a cascata de eventos da ECN, existindo relatos que o tratamento antenatal com betametasona teria efeito na maturação da função motora intestinal e não apenas na inibição da resposta inflamatória. 25

O leite materno exerce uma forte ação seletiva sobre a microflora e é o melhor exemplo de como a dieta pode influenciar a microbiota e como esse ecossistema pode atuar na saúde e nos processos fisiológicos do indivíduo. A partir de dez dias de vida, o intestino da maioria dos $\mathrm{RN}$ a termo é colonizado com flora bacteriana heterogênea, predominando bifidobactérias naqueles alimentados com leite humano, tornando-se diversificada, sem predomínio de nenhuma espécie nas crianças alimentadas com fórmulas. As bifidobactérias são mais tolerantes ao $\mathrm{pH}$ baixo intestinal dos bebês amamentados. Atribui-se aos oligossacarídeos componentes do leite materno o papel bifidogênico mais potente. ${ }^{26-30} \mathrm{O}$ uso de leite materno tem sido advogado como uma forma de prevenir ECN. Barlow et al. ${ }^{31}$ demons-traram, há mais de 30 anos, que o leite materno teve efeito protetor para ECN em roedores. Entretanto, observou-se, posteriormente, a ocorrência de ECN em crianças em aleitamento materno exclusivo, principalmente em leites refrigerados ou pasteurizados. Alguns investigadores sugerem que o uso de leite materno reduz a gravidade da ECN.16,31-33 O maior risco de ECN em crianças alimentadas exclusivamente com fórmulas foi demonstrado por Lucas e Cole 26 em um estudo multicêntrico com uma amostra representativa.

Não foi observado, na população estudada, crianças que usaram apenas fórmula durante todo o período de internação, por isso utilizou-se o uso exclusivo de fórmula por mais de um dia como um indicador de uma dieta de risco para a ECN.

A população estudada apresentou uma incidência 
de 5,3\% para ECN sem nenhuma tendência de incremento na ocorrência desse desfecho e, contrapondose ao aumento de reconhecidos fatores de risco para essa doença nos cinco anos estudados, como uso de antibióticos e NPT, observou-se melhor cobertura de corticoide antenatal, aumento na utilização de leite

\section{Referências}

1. Lin PW, Nasr TR, Stoll BJ. Necrotizing enterocolitis: recent scientific advances in pathophysiology and prevention. Semin Perinatol. 2008;32: 70-82.

2. Grave GD, Nelson SA, Walker WA, Moss RL, Dvorak BV, Hamilton FA, Higgins R, Raju TN. New therapies and preventive approaches for necrotizing enterocolitis: report of a research planning workshop. Pediatr Res. 2007; 62: 510-14.

3. Martin CR, Walker WA. Intestinal immune defences and the inflammatory response in necrotizing enterocolitis. Semin Fetal Neonatal Med. 2006; 11: 369-77.

4. Lee JS, Polin RA. Treatment and prevention of necrotizing enterocolitis. Sem Neonatol. 2003; 8: 449-59.

5. Bell EF. Preventing necrotizing enterocolitis: what works and how safe?. Pediatrics. 2005;115: 173-80.

6. Claud EC, Walker WA. Hypothesis: inappropriate colonization of the premature intestine can cause neonatal necrotizing enterocolitis. FASEB J. 2001; 15: 1398- 403.

7. Boccia D, Stolfi I, Lana S, Moro ML. Nosocomial necrotizing enterocolitis outbreaks: epidemiology and control measures. Eur J Pediatr. 2001; 160: 385-91.

8. Rotbart HA, Levin MJ. How contagious is necrotizing enterocolitis? Pediatr Infect Dis. 1983; 2: 406-13.

9. Meizen-Derr J, Ardythe LM, Hornung RW, Donovan EF, Dietrich KN, Succop PA. Epidemiology of necrotizing enterocolitis temporal clustering in two neonatology practices. J Pediatr. 2009; 154: 656-61.

10. Walsh MC, Kliegman RM. Necrotizing enterocolitis: treatment based on staging criteria. Pediatr Clin North Am. 1986; 33: 179-201.

11. Ballard JL, Khoury JC, Wedig K, Wang L, Eilers-Walsman BL, Lipp R. New Ballard Score, expanded to include extremely premature infants. J Pediatr. 1991;119: 417-23.

12 Guthrie SO, Gordon PV, Thomas V, Thorp JA, Peabody J, Clark RH. Necrotizing enterocolitis among neonates in the United States. J Perinatol. 2003; 23: 278-85.

13. Lemons JA, Bauer CR, Oh W, Korones SB, Papile LA, Stoll BJ, Verter J, Temprosa M, Wright LL, Ehrenkranz RA, Fanaroff AA, Stark A, Carlo W, Tyson JE, Donovan EF, Shankaran S, Stevenson DK. Very low birth weight outcomes of the National Institute of Child Health and Human development neonatal research network, 1995 1996. Pediatrics. 2001; 107: 1-8.

14. Rede Brasileira de Pesquisa Neonatal. Relatório 2010. [Acesso em 3 mar 2012]. Disponível em: http://www.redeneonatal.fiocruz.br/

15. Braga TD, Silva GAP, Lima MC. Revendo a enterocolite necrosante. PRORN. 2011; 8 (4): 119-143. materno exclusivo e redução no consumo de fórmula. Mais estudos são necessários para melhor avaliar a contribuição dessas medidas na não progressão da incidência da ECN nessa população.
16. Neu J, Douglas-Escobar M. Necrotizingenterocolitis: pathogenesis, clinical care and prevention. In: Neu J, Polin RA, editors. Gastroenterology and Nutrition: Neonatology questions and controversies. Philadelphia: Saunders; 2008. p. 281-91.

17. Nanthakumar NN, Fusunyan RD, Sanderson I, Walker WA. Inflamation in the developing human intestine: a possible pathophysiologic contribution to necrotizing enterocolitis. Proc Natl Acad Sci. 2000; 97: 6043-8.

18. Martin CR, Walker WA. Intestinal immune defences and the inflammatory response in necrotizing enterocolitis. Semin Fetal Neonatal Med. 2006; 11: 369-77.

19. Brandt KG, Sampaio MMSC, Miuki CJ. Importância da microflora intestinal. Pediatria. 2006; 28: 117-27.

20 Kalliomäki MA, Walker WA. Physiologic and pathologic interactions of bacteria with gastrointestinal epithelium. Gastroenterol Clin North Am. 2005; 34: 383-99.

21. Walker WA. Development of the Intestinal mucosal Barrier. J Pediatr Gastroenterol Nutr. 2002; 34: 33-9.

22. Walker WA, Goulet O, Morrelli Lorenzo, Antoine JM. Progress in the science of probiotics: from cellular microbiology and applied immunology to clinical nutrition. Eur J Nutr. 2006; 45 (Suppl. 1): 1-18.

23. Lawrence G, Bates J, Gaul A. Pathogenesis of neonatal necrotizing enterocolitis. Lancet. 1982; 319 (8264): 137-9.

24. Lan JG, Cruickshank SM, Singh JCI, Farrar M, Lodge JP, Felsburg PJ, Carding SR. Diferent cytokine response of primary colonic epithelial cells to comensal bacteria. World J Gastroenterol. 2005; 11: 3375-84.

25. DiLorenzo C, Hyman PE. Gastrointestinal motility in neonatal and pediatric practice. Gastroenterol Clin North Am. 1996; 25: 203-24.

26. Lucas A, Cole TJ. Breast milk and neonatal necrotizing enterocolitis. Lancet. 1990; 336 (8730): 1519-23.

27. Sakata H, Yoshioka H, Fujita K. Development of the intestinal flora in very low birth weight infants compared to normal full-term newborns. Eur J Pediatr. 1985; 144: 18690.

28. Gewolb IH, Schwalbe RS, Taciak VL, Harrison TS, Panigrahi P. Stool microflora in extremely low birthweight infants. Arch Dis Child Fetal Neonatal. 1999; 80: F167-73.

29. Hall MA, Cole CB, Smith SL, Fuller R, Rolles CJ. Factors influencing the presence of faecal lactobacilli in early infancy. Arch Dis Child. 1990; 65: 185-8.

30. Blakey JL, Lubitz L, Barnes GL. Development of gut colonization in pre-term neonates. J Med Microbiol. 1982; 15 : 519-29. 
31. Barlow B, Santulli TV, Heird WC, Pitt J, Blanc WA, Schullinger JN. An experimental study of acute neonatal enterocolitis: the importance of breast milk. J Pediatr Surg. 1974; 9: 587-95.

32. Dvorak B, Halpern MD, Holubec H, Dvorakova K, Dominguez JA, Williams CS, Meza YG, Kozakova H,
McCuskey RS. Maternal milk reduces severity of necrotizing enterocolitis and increase intestinal IL-10 in a neonatal rat model. Pediatr Res. 2003; 53: 426-33.

33. Neu J, Walker A. Necrotising enterocolitis. N Engl J Med. 2011; 364: 255-64.

Recebido em 18 de outubro de 2011

Versão final apresentada em 12 de março de 2012

Aprovado em 25 de abril de 2012 